\title{
Diferimiento quirúrgico en pacientes con colecistitis litiásica aguda
}

\author{
Surgical deferral in patients with acute lithiasic cholecystitis
}

\author{
Guadalupe E. Gavilánez-Chávez ${ }^{*}$, Sandra Aguilasocho-Espinoza², Manuel A. Alonso-Lara', \\ Margarito Vega-Vélez', Luz R. Rodríguez-Rivera', David A. Corona-Correa ${ }^{1}$, Luis A. Pulido', \\ Ana M. Sandoval-Moreno y Arnulfo H. Nava-Zavala ${ }^{3}$
}

${ }^{1}$ Servicio de Urgencias, Hospital General Regional No. 46, Instituto Mexicano del Seguro Social (IMSS); ${ }^{2}$ Unidad de Terapia Intensiva, Centro Médico Nacional de Occidente, Instituto Mexicano del Seguro Social (IMSS); ${ }^{3}$ Unidad de Investigación Biomédica 02, Centro Médico Nacional de Occidente, Instituto Mexicano del Seguro Social (IMSS). Guadalajara, Jalisco, México

\section{Introducción}

La colecistitis litiásica es la inflamación de la vesícula biliar ocasionada principalmente por cálculos y lodo biliar. El cólico biliar es la manifestación clínica más habitual, con signo de Murphy positivo ${ }^{1}$. La colecistitis litiásica aguda representa el $9 \%$ del total de los pacientes con síntomas abdominales agudos que acuden a un servicio de urgencias ${ }^{1,2}$. En el $50 \%$ de los pacientes, los episodios de dolor reaparecen tras una primera crisis de dolor biliar ${ }^{3}$. El diferimiento quirúrgico es la postergación de un tratamiento quirúrgico definitivo por causas médicas, del paciente o administrativas. Aproximadamente un $30 \%$ de los pacientes egresados con tratamiento médico reingresan por persistir con la sintomatología, y el porcentaje de vesículas biliares cicatriciales y fibrosas al momento de la cirugía es muy alto, por lo que diferir la cirugía hace que esta sea más difícil e insegura ${ }^{4}$. El tratamiento estándar es la colecistectomía laparoscópica, pero se discute cuál es el mejor momento para su realización: operar en forma precoz o diferir 6-8 semanas la cirugía para operar sin inflamación local. La tendencia predominante es realizar la cirugía precoz, pues numerosos estudios la muestran como factible y segura ${ }^{4}$; sin embargo, en nuestro centro de trabajo se opta por el diferimiento quirúrgico, por lo que nuestro objetivo fue describir las complicaciones por colecistitis litiásica relacionadas con el diferimiento quirúrgico en pacientes del servicio de urgencias.

\section{Método}

Estudio descriptivo transversal con revisión de 143 expedientes de pacientes con colecistitis litiásica aguda con dos o más reingresos de enero a diciembre de 2018. El análisis estadístico se realizo con el programa Excel mediante estadística descriptiva.

\section{Resultados}

Se evaluaron 456 casos de colecistitis aguda, de los cuales 143 fueron incluidos como universo de estudio. De estos, 97 (67.8\%) fueron mujeres y 46 (32.1\%) hombres, y la distribución etaria tuvo una media de $41 \pm 13.7$ años. El tiempo para la realizacion de la colecistectomía fue en promedio de 82 días a partir del diagnóstico, con un rango de 1 a 433 dias. Las causas del diferimiento de la cirugía se describen en la tabla 1.

De los pacientes que fueron diferidos para su intervención, el $24.4 \%$ presentaron alguna complicación, coledocolitiasis 15 (42.85\%), pancreatitis $10(28.57 \%)$, colangitis $5(14.2 \%)$, sepsis $5(14.2 \%)$, hidrocolecisto $5(14.2 \%)$ y choque séptico $5(14.2 \%)$, resultando una muerte por colangitis y choque séptico.

\section{Correspondencia:}

*Guadalupe E. Gavilánez-Chávez E-mail: dragavilanez@gmail.com
Fecha de recepción: 19-11-2021

Fecha de aceptación: 13-12-2021 DOI: 10.24875/REIE.21000156
Disponible en internet: 07-03-2022
Rev Educ Investig Emer. 2022;4(Supl 1):27-28
www.medicinadeemergencias.com www.medicinadeemergencias.com bajo la licencia CC BY-NC-ND (http://creativecommons.org/licenses/by-nc-nd/4.0/). 
Tabla 1. Causa de diferimiento de la cirugía en 143 pacientes con colecistitis litiásica aguda

\begin{tabular}{|l|r|c|}
\hline Causa & n & $\%$ \\
\hline «No urgencia quirúrgica» & 32 & 22.37 \\
\hline Falta de tiempo quirúrgico & 32 & 22.37 \\
\hline $\begin{array}{l}\text { Negación a firma de consentimiento informado } \\
\text { Inestabilidad hemodinámica con alto riesgo }\end{array}$ & 7 & 4.89 \\
\hline $\begin{array}{l}\text { quirúrgico } \\
\text { Sin explicación del diferimiento en el expediente }\end{array}$ & 53 & 13.28 \\
\hline
\end{tabular}

\section{Conclusiones}

El tiempo entre el primer episodio de dolor y la realización de la cirugía es prolongado. Se deben encontrar estrategias conjuntas médico-administrativas para reducirlo y con ello disminuir las posibilidades de complicación.

\section{Financiamiento}

El financiamiento para la realización del presente trabajo fue asumido por los autores.

\section{Conflicto de intereses}

Los autores declaran no tener conflictos de intereses.

\section{Responsabilidades éticas}

Protección de personas y animales. Los autores declaran que para esta investigación no se han realizado experimentos en seres humanos ni en animales.

Confidencialidad de los datos. Los autores declaran que en este artículo no aparecen datos de pacientes.

Derecho a la privacidad y consentimiento informado. Los autores declaran que en este artículo no aparecen datos de pacientes.

\section{Bibliografía}

1. Cirino E, Bonfiglio S, Buffone A, Alberghina F, Basile G, Intelisano G. La colecistite acuta. Chir Ital. 1984;36:1046-64.

2. Chawla A, Bosco JI, Lim TC, Srinivasan S, Teh HS, Shenoy JN. Imaging of acute cholecystitis and cholecystitis-associated complications in the emergency setting. Singapore Med J. 2015;56:438-43.

3. European Association for the Study of the Liver (EASL). EASL Clinical Practice Guidelines on the prevention, diagnosis and treatment of gallstones. J Hepatol. 2016;65:146-81.

4. López J, Iribarren O, Hermosilla R, Fuentes T, Astudillo E, López N, et al. Resolución quirúrgica de la colecistitis aguda. ¿Influye el tiempo de evolución? Rev Chil Cir. 2017;69:129-34. 